\title{
Abstract from Current Literature
}

Treatment of steroid-resistant nephrotic syndrome in children: new guidelines from KDIGO

Rebecca M. Lombel \& Elisabeth M. Hodson \& Debbie S. Gipson

Pediatr Nephrol (2013) 28:409-414

Kidney Disease: Improving Global Outcomes (KDIGO) recently published the clinical practice guidelineon glomerulonephritis (GN) to assist the practitioner caring for patients with GN. Chapter 4 of the guideline focuses on managing children aged 1-18 years with steroidresistant nephrotic syndrome (SRNS), defined by an inability to achieve complete remission with corticosteroid therapy. Guideline development followed a thorough evidence review, and management recommendations and suggestions were based on the best available evidence. Limitations of the evidence, including the paucity of large-scale randomized controlled trials, are discussed. This article provides both the guideline recommendations and a brief review of relevant treatment trials related to each recommendation.

This précis serves as a summary of the complete guidelines recently published.

Urine biomarkers in juvenile-onset SLE nephritis Louise Watson \& Michael W. Beresford

Pediatr Nephrol (2013) 28:363-374

Over $80 \%$ of patients with juvenile-onset systemic lupus erythematosus will have renal involvement compared to

$40 \%$ with adult-onset disease. Up to $44 \%$ of children who do have lupus nephritis (LN) progress to renal failure in early adulthood. Improved methods of detecting onset of LN would allow earlier treatment, which may prevent irreversible renal scarring and a decline in renal function. Current conventional markers of disease activity fail to adequately predict renal lupus flares and include proteinuria, complement levels, antidouble-stranded DNA antibodies and serum creatinine concentrations. Standardized histological classification is currently the gold standard for diagnosing and classifying LN, but its invasive nature limits routine use for monitoring, especially in a childhood population. Novel biomarkers need to be sensitive and specific - and preferably non-invasive and cost-effective. The most promising biomarkers in juvenileonset $L N$ include urinary neutrophil gelatinase associated lipocalin, monocyte chemoattractant protein 1 and transforming growth factor-beta, although many others have been identified and are under investigation. No one biomarker yet discovered is unique to $L N$, indicating an overlap in disease pathophysiology. It is likely that a combination of biomarkers will be required for assessing disease flare detection, response to treatment and prognostic information. Potential biomarkers require longitudinal validation in large paediatric, prospective cohorts to assess their ability to act as clinically useful adjuncts.

Neonatal anthropometrics and correlation to childhood obesity-data from the Danish Children's Obesity Clinic

Ulrik Lausten-Thomsen \& Dorthe Sadowa Bille \& Ida Nässlund \& Lise Folskov \& Torben Larsen \& JensChristian Holm

Received: 14 November 2012 / Accepted: 14 January 2013 \# Springer-Verlag Berlin Heidelberg 2013

Eur J Pediatr. Published Online: 01 February 2013

Recent evidence has demonstrated the prenatal initiation of childhood obesity as epidemiological studies and animal studies have illustrated the effect of the intrauterine milieu for subsequent development of childhood obesity. This study investigates the relationship between severe childhood obesity and the preceding in utero conditions expressed by birth weight and birth length, birth weight- for-gestationalage and neonatal ponderal index in a Danish cohort of 1,171 severely obese children (median age 11.48 years, range 3.13 to 17.98 years) with a mean body mass index-standard derivation score (BMI-SDS) of +2.96 (range +1.65 to +9.72 ) treated in our national referral centre. In a linear general regression model adjusted for socioeconomic status and breastfeeding duration, a significant linear correlation between BMISDS at time of enrolment and both birth weight $(p$, 
$3.8 \times 10 " 6)$ and birth length ( $p, 6.1 \times 10 " 4)$, birth-weightfor-gestational age $(p, 4.3 \times 10 " 7)$ and the neonatal ponderal index $(p, 0.02)$ was demonstrated. Duration of breastfeeding, however, was not found to be significant for either the BMI-SDS/BW or the BMISDS/BL correlation. Conclusion: These results indicate that the prenatal period can be considered as a potential window of opportunity for prevention of childhood overweight and obesity and anthropological measurements may in theory be used to help identify neonates at high risk for developing childhood obesity.

\section{Lactobacillus reuteri DSM 17938 for the Management of Infantile Colic in Breastfed Infants: A Randomized, Double-Blind, Placebo- Controlled Trial}

Hania Szajewska, MD Ewa Gyrczuk, MD Andrea Horvath, MD

The Journal of Pediatrics Volume 162, Issue 2 , Pages 257-262, February 2013

Objective: To determine whether administration of Lactobacillus reuteri (L reuteri) DSM 17938 is beneficial in breastfed infants with infantile colic.
Study design: Eighty infants aged $<5$ months with infantile colic (defined as crying episodes lasting 3 or more hours per day and occurring at least 3 days per week within 7 days prior to enrollment), who were exclusively or predominantly $(>50 \%)$ breastfed were randomly assigned to receive $L$ reuteri DSM 17938 ( $10^{8}$ colony-forming units) $(n=40)$ or an identically appearing and tasting placebo $(n=40)$, both orally, in 5 drops, 1 time daily, for 21 days. The primary outcome measures were the treatment success, defined as the percentage of children achieving a reduction in the daily average crying time e" $50 \%$, and the duration of crying (minutes per day) at $7,14,21$, and 28 days after randomization.

Results: The rate of responders to treatment was significantly higher in the probiotic group compared with the placebo group at day $7(P=.026)$, at day 14 (relative risk (RR) 4.3, 95\% Cl 2.3-8.7), at day 21 (RR $2.7,95 \% \mathrm{Cl} 1.85-4.1)$, and at day 28 (RR $2.5,95 \% \mathrm{Cl}$ 1.8-3.75). In addition, throughout the study period, the median crying time was significantly reduced in the probiotic group compared with the control group.

Conclusion : Exclusively or predominantly breastfed infants with infantile colic benefit from the administration of $L$ reuteri DSM 17938 compared with placebo. 